\title{
EXHAUSTIVIDAD DE LA NOTIFICACIÓN DE LOS CASOS DE SIDA EN CATALUÑA: UN ESTUDIO BASADO EN LAS SOLICITUDES DE DETERMINACIONES DE LINFOCITOS CD4 Y EN LA PRESCRIPCIÓN DE ANTIRRETROVIRALES
}

\author{
Patricia García de Olalla Rizo (1), Martí Vall Mayans (2), Montserrat Miret Mases (2), Roser Clos \\ Guix (1), Jordi Casabona Barbarà (2), Joan A. Caylà Buqueras (1), y Grupo Colaborador *. \\ (1) Servei d'Epidemiologia, Institut Municipal de Salut Pública, Barcelona. \\ (2) Centre d'Estudis Epidemiològics sobre la SIDA de Catalunya, Badalona (CEESCAT). \\ (*) Grupo Colaborador: Josep Ribas, Josep Mallolas (Hospital Clinic i Provincial), José Luis Rodríguez (Hospital \\ Santa Creu i Sant Pau), Teresa Español (Hospital Gral. Vall d'Hebron), Josep Monterde, Santiago Grau (Hospital \\ Ntra Sra del Mar), Enric Buendía, Mercè Corominas (Ciutat Sanitaria de Bellvitge, L'Hospitalet), Ricardo Pujol, \\ Bonaventura Clotet (Hospital Germans Trias i Pujol, Badalona), Carmen Alonso, María Jesús Gallart (Hospital \\ Joan XXIII, Tarragona).
}

\section{RESUMEN}

Fundamento: Determinar la tasa de notificación de SIDA en Cataluña.

Método: Se utilizan como fuentes de contraste las solicitudes de las determinaciones de linfocitos T CD4+ y la prescripción de antirretrovirales en siete hospitales universitarios de Cataluña entre el 1 de enero y el 31 de junio de 1994. Se consideró que un caso no estaba declarado cuando en la historia clínica existía un diagnóstico de sida (según la definición de Europa 93) y dicho caso no se encontraba incluido en el registro de sida de Cataluña.

Resultados: De los 1.370 casos analizados, se detectaron 50 casos de sida no declarados. La tasa de notificación global fue del 98,6\%, con un intervalo de confianza del 95\% (IC del 95\%):98,299,0. La mayoría de los casos no declarados eran hombres (72\%) con una edad media de 37,3 años (DS:11,8), el 52\% eran usuarios de drogas por vía parenteral (UDVP), siendo la tuberculosis extrapulmonar la enfermedad diagnóstica más frecuente (16\%), el $81,4 \%$ presentaban un nivel de linfocitos T CD4+ menor o igual a 200 células $/ \mathrm{mm}^{3}$ El $92 \%$ de estos casos se detectaron a partir de los registros de determinaciones de linfocitos T CD4+.

Conclusiones: La exhaustividad detectada se considera adecuada. Se recomienda usar los registros de determinaciones linfocitarias para este tipo de estudios.

Palabras clave: Vigilancia. Sida. Subnotificación. CD4+. Terapia antirretroviral.

Correspondencia:

Patricia G de Olalla

Instituto Municipal de Salud Pública

Plaça Lesseps, 1

08023 Barcelona

Correo electrónico: polalla @imsb.bcn.es

\section{ABSTRACT \\ The Degree to which all Existing Aids Cases are Officially reported in Catalunya: a Study Based on the Requests for CD4 White Blood Cell Counts and the Prescribing of Antitretroviral Drugs}

Background: To ascertain the degree to which AIDS is officially reported in Catalunya.

Method: The request for T CD4+ white blood cell counts and the prescribing of antiretroviral drugs in seven university hospitals in Catalunya from January 1, 1994 to June 31, 1994 were used as data-comparison sources. A case was considered to not have been reported when the clinical history showed a diagnosis of AIDS (according to the Europe 93 definition) and the case in question did not show up on the AIDS Registry for Catalunya.

Results: Of the 1,370 cases studied, 50 unreported AIDS cases were found. In all, $98.6 \%$ of all cases were found to have been reported, for a $95 \%$ confidence interval $(95 \% \mathrm{CI}): 98.2-99.0$. Most of the unreported cases were males $(72 \%)$ averaging 37.3 years of age (HCA: 11.8), 52\% of whom were injected drug users (IDU's), extrapulmonary tuberculosis being the disease most often diagnosed $(16 \%), 81.4 \%$ having been found to have a T CD4+ white blood cell count of $200 \mathrm{WBC} / \mathrm{mm}^{3}$ or below. $92 \%$ of these cases were detected based on T CD4+ white blood cell counts.

Conclusions: The degree of completeness found is considered to be adequate. It is recommended that the white blood cell count records being used for this type of studies.

Key words: Surveillance. AIDS. Underreporting. CD4 counts. Antiretroviral therapy. 


\section{INTRODUCCIÓN}

La vigilancia epidemiológica (VE) del síndrome de inmunodeficiencia adquirida (sida) tiene por objeto describir las características y la evolución de esta epidemia, así como evaluar el impacto de la morbilidad y la mortalidad relacionada con el virus de la inmunodeficiencia humana (HIV). La utilidad de la información procedente de la VE depende tanto del grado de exhaustividad alcanzado en la notificación de los casos, como de la calidad obtenida y, debido a que ésta es utilizada por clínicos, epidemiólogos y planificadores de la salud, es de suma importancia que sea lo más completa y válida posible' ${ }^{1}$.

La notificación de los casos de sida en Cataluña es obligatoria desde el año 1986 y se coordina a través del Centro de Estudios Epidemiológicos sobre el sida de $\mathrm{Ca}$ taluña (CEESCAT), vinculado al Departamento de Sanidad y Seguridad Social (DSSS $)^{2}$. El Registro de casos recoge de manera sistemática los casos de sida detectados por el Instituto Municipal de Salud Pública en la ciudad de Barcelona, de forma activa, a partir de los datos procedentes de diferentes fuentes (sistema de enfermedades de declaración obligatoria, altas hospitalarias, registro de mortalidad, registro de tuberculosis y registro de drogodependencias). En el resto de Cataluña son las delegaciones territoriales del DSSS, la dirección general de Servicios Penitenciarios y de Rehabilitación del Departamento de Justicia y, provisionalmente, la región sanitaria «Centre» del Servicio Catalán de la Salud los encargados de recoger las notificaciones de los casos de SIDA y tramitarlas al CEESC $\Lambda$ T.

El objetivo del presente estudio es determinar el grado de exhaustividad de la notificación de casos de sida en Cataluña a partir del recuento de linfocitos T CD4+ y de la prescripción de antirretrovirales.

\section{SUJETOS Y MÉTODOS}

El estudio se efectuó en siete hospitales universitarios (cuatro de la ciudad de Barcelona; dos de la provincia de Barcelona; y uno de la ciudad de Tarragona). Después de la revisión manual o informática de las historias clínicas de los pacientes atendidos entre enero y junio de 1994 a los que se les había realizado al menos una determinación de linfocitos T CD4+, y ésta hubiera sido menor o igual a 500 células $/ \mathrm{mm}^{3}$ (porcentaje menor o igual a $29 \%$ ), y/o a los que se les hubiera prescrito algún tipo de terapia antirretroviral en la misma época ${ }^{3}$. Una vez excluidos los pacientes no infectados por VIH, se eliminaron los casos duplicados, los menores de 15 años y aquellos que ya estaban incluidos en el Registro de sida. Seguidamente, se procedió a la revisión de las historias clínicas de los pacientes VIH positivos que se incluyeron en el estudio, recogiéndose mediante un cuestionario estructurado diferentes variables demográficas y epidemiológicas (fecha de nacimiento, sexo, lugar de residencia, mecanismo de transmisión del VIH), variables clínicas y terapéuticas (tratamiento antirretroviral, determinación de linfocitos $\mathrm{T}$ $\mathrm{CD} 4+$, enfermedad indicativa de SIDA), así como, centro y fecha de diagnóstico, y fecha del último control en caso de no haber sido diagnosticado aún de sida.

Sólo, en un hospital se obtuvieron los datos de las dos fuentes de información. En el resto de los centros no fue factible debido a la dificultad en la identificación de los pacientes, o a la imposibilidad de elaboración de un listado, pues los datos no estaban informatizados. Por razones operativas, en los dos hospitales de la provincia de Barcelona, sólo se pudo realizar la revisión de una muestra aleatoria del $25 \%$ de las historias en uno de ellos y del $33 \%$ en el otro.

Se consideró que un caso no estaba declarado cuando en la historia clínica existía un diagnóstico de sida (según la definición de Europa 93 $)^{5}$ con anterioridad al 1 de julio de 1994 y éste no estaba incluido en el Registro 
de sida. La revisión y contraste con los casos del Registro se realizó entre noviembre de 1995 y julio de 1997.

Para cada centro participante se calculó la tasa de notificación observada (TO), cuyo numerador eran los casos declarados (R) durantc los años 1991, 1992, 1993 y 1994 , siendo el denominador el número de casos declarados (R) más el número de casos diagnosticados de sida y que no habían sido declarados (nD). Así, $\mathrm{TO}=\mathrm{R} * 100 /(\mathrm{R}+\mathrm{nD})$. Se calculó, también, la tasa de notificación estimada (TE) que tenía en cuenta el número de casos presumiblemente no declarados (nD2) correspondientes a aquellos pacientes en los que los datos disponibles no permitían asegurar o descartar el diagnóstico de sida. Estos casos se calcularon aplicando la misma proporción de casos no declarados encontrada en las historias revisadas. Así, $\mathrm{TE}=\mathrm{R}^{*} 100 / \mathrm{R}+(\mathrm{nD}+\mathrm{nD} 2)$. En ambos casos, se calcularon los intervalos de confianza del 95\% (IC del 95\%). En la comparación de la tasas de utilizó la prueba del ji cuadrado o la prueba exacta de Fisher en caso de valores $<5$.

Para cada centro se realizó un análisis descriptivo de las variables recogidas, así como un análisis comparativo entre el grupo de casos en los cuales se conocía si tenían sida o no y aquellos en los que se desconocía esta información. En el análisis estadístico se utilizó el test de la $\mathbf{t}$ de student para comparar las variables cuantitativas, la prueba del ji cuadrado para las variables categóricas, y como medida de asociación se calcularon Odds ratio (OR), con IC del $95 \%$.

\section{RESULTADOS}

En la tabla 1 se presentan las características descriptivas de los pacientes VIH positivos y las tasas de notificación por centros.

\section{Hospitales de Barcelona}

En el Hospital del Mar, se obtuvo un listado de 117 casos VIH positivos que recibían tratamiento antirretroviral. En el $72 \%$ de los casos $(n=84)$ se pudo realizar el seguimiento hasta la fecha de cierre del estudio, después de la revisión de su historia clínica, se concluyó que no existían casos con diagnóstico de sida.

En el Hospital Clínic se revisaron las historias clínicas de 417 pacientes VIH positivos que recibían tratamiento antirretroviral. En el $15 \%$ de estos no fue posible concluir su estado en relación al sida, pues o no se controlaban regularmente o bien habían dejado de acudir al centro. Entre el $85 \%$ restante $(n=354)$, se detectaron 4 casos diagnosticados de sida que no habían sido declarados.

En el Hospital de Sant Pau se obtuvo un listado del servicio de inmunología de 392 VIH positivos que se habían realizado una determinación de linfocitos T CD4+ durante el período estudiado. El $74 \%$ de estos $(\mathrm{n}=288)$ se pudo revisar, detectándose 17 casos de SIDA no declarados.

De un total de 443 pacientes VIH positivos procedentes del registro del Servicio de Inmunología del Hospital Vall d'Hebrón. Se revisaron el $52 \%$ de los casos $(n=225)$, entre ellos se detectaron 3 casos de SIDA no declarados.

\section{Otros hospitales}

En la Ciudad Sanitaria de Bellvitge, L'Hospitalet, se partió de una muestra aleatoria del $25 \%$ de los 648 pacientes VIH positivos procedentes del registro del Servicio de Inmunología. De los 132 casos revisados había 2 casos de sida no declarados.

En el Hospital Germans Trias i Pujol de Badalona, de los 703 pacientes procedentes del registro del Servicio de Inmunología, se 
Tabla 1

Características descriptivas de los pacientes VIH positivos y tasas de notificación según centro hospitalario

\begin{tabular}{|c|c|c|c|c|c|c|c|c|}
\hline VARIABLES & $\begin{array}{c}\text { H. del Mar } \\
n(\%) \\
84\end{array}$ & $\begin{array}{c}\text { H. Clinic } \\
n(\%) \\
354\end{array}$ & $\begin{array}{l}\text { H. Sant Pau } \\
n(\%) \\
288\end{array}$ & $\begin{array}{c}\text { H. Vall d'Hebron } \\
n(\%) \\
225\end{array}$ & $\begin{array}{c}\text { C. Sanitaria, } \\
\text { Bellvitge } \\
n(\%) \\
132\end{array}$ & $\begin{array}{c}\text { H. G. Trias } i \\
\text { Pujol } \\
n(\%) \\
231\end{array}$ & $\begin{array}{c}\text { H. Joan XXIII } \\
n(\%) \\
56\end{array}$ & $\begin{array}{c}\text { TOTAL } \\
n(\%) \\
1370\end{array}$ \\
\hline \multicolumn{9}{|l|}{ SEXO } \\
\hline hombres & $58(69,0)$ & $245(69,2)$ & $211(73,3)$ & $167(74,2)$ & $111(84,1)$ & $171(74,0)$ & $44(78,6)$ & $1007(73,5)$ \\
\hline mujeres & $26(31,0)$ & $108(30,5)$ & $76(26,4)$ & $58(25,8)$ & $21(15,9)$ & $58(25,1)$ & $12(21,4)$ & $359(26,2)$ \\
\hline desconocido & & $1(0,3)$ & $1(0,3)$ & & & $2(0,9)$ & & $4(0,3)$ \\
\hline Edad años media (DS) & $34(8)$ & $34,9(9)$ & $35,1(7,4)$ & $33,6(7,8)$ & $32.4(7,1)$ & $34,8(9,1)$ & $32,6(5,8)$ & $34,2(8,1)$ \\
\hline \multicolumn{9}{|l|}{ Grupo de riesgo } \\
\hline UDVP & $48(57,1)$ & $127(35,9)$ & $184(63,9)$ & $135(60,0)$ & $95(72,0)$ & $36(15,6)$ & $45(80,4)$ & $670(48,9)$ \\
\hline HMS & $3(3,6)$ & $85(24)$ & $41(14,2)$ & $32(14,2)$ & $13(9,8)$ & $22(9,5)$ & $3(5,3)$ & $199(14,5)$ \\
\hline HMS+UDVP & $2(2,4)$ & $1(0,3)$ & $1(0,3)$ & $1(0,4)$ & $1(0,8)$ & & - & $6(0,4)$ \\
\hline HH & - & $8(2,2)$ & $2(0,7)$ & $2(0,9)$ & $2(1,5)$ & & $2(3,6)$ & $16(1,2)$ \\
\hline HTS & $20(23,8)$ & $36(10,2)$ & $31(10,8)$ & $20(8,9)$ & $14(10,6)$ & $11(4,8)$ & $4(7,1)$ & $139(10,1)$ \\
\hline desconocido & $11(13,1)$ & $97(27,4)$ & $29(10,1)$ & $35(15,6)$ & $7(5,3)$ & $162(70,1)$ & $2(3,6)$ & $340(24,9)$ \\
\hline $\mathrm{CD} 4 \leq 200$ & $35(41,7)$ & & $92(3 I, 9)$ & $69(30,7)$ & $47(35,6)$ & $101(43,7)$ & $18(32,1)$ & $362(26,4)$ \\
\hline $\begin{array}{l}\text { n. de casos } \\
\text { no declarados }\end{array}$ & & $4(1,1)$ & $17(5,9)$ & $3(1,3)$ & $2(1,5)$ & $23(10,0)$ & $1(1,8)$ & $50(3,6)$ \\
\hline Tasa observada & $100 \%$ & $99,6 \%$ & $95,0 \%$ & $99,4 \%$ & $99,7 \%$ & $94,4 \%$ & $99,6 \%$ & $98,6 \%$ \\
\hline IC del $95 \%$ & $99,2-100$ & $99,0-99,9$ & $92,1-97,1$ & $98,3-99,9$ & $98,8-100$ & $91,8-96,4$ & $97,8-100$ & $98,2-99,0$ \\
\hline Tasa estimada & & $99,5 \%$ & $93,3 \%$ & $98,9 \%$ & $98,3 \%$ & $84,8 \%$ & $98,8 \%$ & $96,5 \%$ \\
\hline IC del $95 \%$ & & $98,5-99,8$ & $90,2-95,7$ & $97,5-99,6$ & $97,0-99,2$ & $81,5-88,1$ & $96,6-99,7$ & $95,8-97,0$ \\
\hline
\end{tabular}

* UDVP: usuario de drogas por vía parenteral, HMS: homosexual, HH: hemofítion-hemotransfundido, HTS: heternsexual. 
revisó una muestra de 231 pacientes, lo que aportó 23 casos de sida no declarados.

Finalmente, en el Hospital Joan XXIII de Tarragona se identificaron 188 pacientes VIH positivos, $20 \%$ se habían recogido a través del registro de farmacia, $65 \%$ de hematología y $15 \%$ de ambos registros. La revisión de los datos correspondientes a los 188 casos investigados, permitió concluir que en el $70 \%$ de los casos no constaban controles hospitalarios que permitieran decidir sobre el diagnóstico de sida, ya que no se controlaban en dicho centro, y que en el $30 \%$ restante sólo se detectó 1 caso de sida no declarado.

En relación con las características de los 50 casos de sida no declarados, en su mayoría eran hombres $(72 \%)$, con una edad media de 37,3 años (DS:11,8), el 52\% eran UDVP, siendo la tuberculosis extrapulmonar la enfermedad diagnóstica de sida más frecuente $(16 \%)$, el $81,4 \%$ presentaban un nivel de linfocitos T CD4+ menor o igual a 200 células $/ \mathrm{mm}^{3}$. El $92 \%$ de estos casos provenían de los registros de determinaciones de CD4+.

Al comparar los casos en los que se conocía si tenían o no sida con aquellos en los que esta variable era desconocida, no se observaron diferencias significativas ni con relación al sexo, a la edad, al porcentaje de linfocitos $\mathrm{T} \mathrm{CD} 4+$, pero si en lo que hace referencia al mecanismo de transmisión. En el sentido que en los UDVPs (usuarios de drogas por vía parenteral) era más frecuente concluir si tenían o no sida, presentando OR que variaban entre 9,6 (IC del 95\%:3,1129,95 ) y 59,7 (IC del 95\%:18,42-192,25), según el centro.

Tampoco, se evidenciaron diferencias estadísticamente significativas al comparar las variables anteriormente citadas de los casos no declarados con las de los casos incluidos en el Registro de sida.

Además de las tasas observadas y estimadas presentadas en la tabla 1 , se calcularon las tasas de notificación según el año de diagnóstico (variando esta tasa entre el $97,4 \%$ del año 1994 y el 99,7\% del año 1991), según el sexo (en los hombres se observó una tasa de notificación del $98,8 \%$ y en las mujeres del 97,9\%), y según el mecanismo de transmisión (los UDVPs y el grupo de los homosexuales presentaron una tasa del $98,7 \%$ ), sin llegar a ser en ningún caso la diferencia estadisticamente significativa.

\section{DISCUSIÓN}

El presente estudio es el primero de estas características realizado en España, estimándose que los resultados son satisfactorios para el sistema de VE en los principales hospitales de Cataluña, ya que muestran unas tasas de notificación de casos de sida superiores al $90 \%$ en el año 1994. Esta tasa es similar $(92 \%)$ a la encontrada de forma indirecta en un estudio realizado en Cataluña ${ }^{6}, y$ en otros realizados fuera de España ${ }^{7,8}$.

Los resultados de los estudios de notificación no son homogéneos entre ciudades de un mismo país ${ }^{9.10}$, creyéndose que pucda existir subnotificación específica para determinados grupos de transmisión, aunque no hay un patrón claro, con excepción, quizás, del grupo de transmisión heterosexual y vertical $^{11}$, hecho que no se ha podido constatar en este estudio. Por otra parte, este estudio ha puesto de manifiesto que los casos de pacientes VIH positivos, cuyo mecanismo de transmisión era la vía parenteral, se controlaban con más asiduidad que el resto de los grupos.

La aproximación que se ha seguido en este trabajo de investigación es laboriosa ya que existen serias limitaciones a nivel informático en algunos de los centros asistenciales investigados, lo que dificulta obtener de forma ágil datos con las variables de interés. Dejando aparte este aspecto metodológico, no es probable que las tasas observadas en este estudio estén sujetas a algún tipo de sesgo, ya que éstas son bastante parecidas 
entre centros, oscilando entre el $91,8 \%$ y el $100 \%$, siendo distintas, no obstante, la titularidad y las prácticas de la organización de la asistencia de los enfermos con sida en dichos centros. Por otro lado, entendemos que durante los últimos años tampoco hay ningún dato que haga pensar en que la tasa de notificación haya disminuido, y que la disminución de los casos de sida en $1997^{12}$ se debe - sobre todo- al efecto de las terapias antirretrovirales ${ }^{13}$ y no a la falta de notificación.

Los cambios de definición de sida han avanzado el diagnóstico del sida en pacientes con un nivel de linfocitos T CD4+ aún conservado, como es el caso de la tuberculosis pulmonar. Esto ha facilitado la participación de los centros extrahospitalarios en la notificación de los $\operatorname{casos}^{14,15}$, los cuales, según datos de Estados Unidos, podrían presentar niveles más bajos de notificación que los centros hospitalarios ${ }^{16}$. Este, quizás, sea un dato a valorar en nuestro contexto, ya que los resultados del estudio que presentamos se basa en datos hospitalarios exclusivamente. En cualquier caso, la implantación de métodos de vigilancia epidemiológica activa a todos los niveles es la única que puede garantizar unos niveles óptimos de exhaustividad de los casos de sida ${ }^{17.18}$. Es muy probable que en 1998, debido a los cambios espectaculares en el curso de la infección HIV a raíz de la introducción generalizada de los tratamientos antirretrovirales combinados, la notificación de los casos de sida sea manifiestamente insuficiente para obtener una imagen real del curso de la epidemia HIV. Como ejemplo, nuestro estudio estimó que al menos un $30 \%$ de casos adicionales habrían sido notificados si se hubiera aplicado sólo el criterio del CDC de 1992 basado en un número de linfocitos CD4 inferior $200 / \mathrm{mm} 3$. Es por esto que los sistemas de VE deben modernizarse en la recogida y en la diseminación de la información ${ }^{19}$ para adaptarse y, preferiblemente, anticiparse al curso real de la epidemia del HIV.
Los datos aquí presentados son aplicables sólo a Cataluña, ya que las prácticas de notificación no son homogéneas en el Estado español.

En definitiva, la exhaustividad del registro de sida se considera adecuada, aunque debe de ser revisada periódicamente (basándose prioritariamente en las determinaciones de CD4, por ser más efectiva). Además, si en un futuro inmediato se implementa un sistema de declaración de la propia infección por $\mathrm{VIH}^{20}$, deberá diseñarse de tal forma que facilite el mantenimiento del grado de notificación de los casos de SIDA actual, pues este parámetro seguirá siendo importante tanto para evaluar la efectividad de los tratamientos antirretrovirales, así como para permitir comparaciones entre diferentes países.

\section{AGRADECIMIENTOS}

A todos los médicos que han colaborado en la vigilancia epidemiológica.

\section{BIRI IOGRAFÍA}

1. Evans BG. Estimating underreporting of AIDS: straightforward in theory-difficult in practice. AIDS 1991;5:1261-2.

2. CEESCAT. Sistema Integrat de Vigilància Epidemiològica del VIH/SIDA a Catalunya (SIVES). Informe Anual 1996. Badalona: Departament de Sanitat i Seguritat Social; 1997.

3. Buehler JW, Berkelman RL, Sther-Green JK. The completeness of AIDS surveillance. J Acquir Immune Defic Syndr 1992;5:257-64.

4. Centers for Disease Control. Revision of the CDC surveillance case definition for acquired immunodeciciency syndrome. Morbidity and Mortality Weekly Report 1987;36:36:n. ${ }^{\circ} 1 \mathrm{~S}$.

5. Ancelle-Park R. Expanded European AIDS case definition. Lancet 1993;341:441.

6. Mingot M, Salas T, Segura A, Casabona J. Mejora de las estadísticas de mortalidad por SIDA en Cataluña. Gac Sanit 1994;8:122-7. 
7. Hickman M, Aldous J, Gazzard B, Ellam A. AIDS surveillance: a direct assessment of underreporting. AIDS 1993;7:1661-5.

8. Rosenblaum L, Buehler JW, Morgan MW, Costa S, Hidalgo J, Holmes R, Lieb L, Shields A, Whyte BM. The completeness of AIDS case reporting, 1988: a multisite collaborative surveillance project. Am J Public Health 1992;82:1495.

9. Fife D, MacGregor RR, McAnaney. Limitations of AIDS reporting under favorable circumstances. Am J Prev Med 1993:9 (5):317-20.

10. Conway GA, Colley-Niemeyer B, Pursley C, Cruz C, Burt S, Rion P, Heath CW. Underreporting of AIDS cases in South Carolina, 1986 and 1987. JAMA 1989;262:2859-63.

11. Centre Européen pour la Surveillance Épidémiologique du SIDA. Surveillance du VIH/SIDA en Europe: Rapport Trimestriel n. ${ }^{\circ}$ 49. mars 1996.

12. Anónimo. Vigilància epidemiològica de la sida a Catalunya. Situació fins al 31 de desembre de 1997.Bul Epidemiol de Catalunya 1998;19:1116.

13. Palella FJ, Delaney KM, Moorman AC, Loveless MO, Fuhrer J, Satten GA, et al. Declining Morbidity and Mortality among Patients with advanced Human Immunodeficiency Virus Infection. N Engl J Med 1998;338:853-860.
14. CEESCAT Vigilància epidemiològica de la sida. Situació fins a 31 de desembre de 1997. Butlletí Epidemiològic de Catalunya 1998; (en prensa).

15. García de Olalla $P$, Caylà JA, Jansà JM, GaldósTangüis H, Brugal MT, Pañella H, Maldonado R. SIDA a Barcelona. Vigilància epidemiològica. Situació a 31 de desembre de 1997. Informe Núm. 38.

16. Bernillon $\mathrm{P}$, Lièvre L, Pillonel J, Laporte A, Costagliola D. Estimation de la sous-déclaration des cas de SIDA en France par le méthode de capture-recapture. Bull Épidemiol Hebdomadaire 1997; Núm spécial Novembre 1997:54-55.

17. Chamberland ME, Allen JR, Monroe JM, García N, Morgan C, Reiss R, Stephens H, Walker J, Friedman SM. Acquired immunodeficiency syndrome in New York city. Evaluation of an active surveillance system. JAMA 1985;254:383-7.

18. Trino R, McAneny J, Fife D. Laboratory-based reporting of AIDS. J Acquir Immune Defic Syndr 1993;6:1057-61.

19. Greenspan AL and Curran JW. Communicating surveillance, epidemiologic, and laboratory information on HIV infection and AIDS. Public Health Rep 1991;106:721-6.

20. European Centre for the Epidemiological monitoring of AIDS. HIV/AIDS Surveillance in Europe: Quarterly Report no.56, 31 December 1997. 